\title{
Convergence of Rothe's Method for Fully Nonlinear Parabolic Equations
}

\author{
By Ivan Blank and Penelope Smith
}

\begin{abstract}
Convergence of Rothe's method for the fully nonlinear parabolic equation $u_{t}+$ $F\left(D^{2} u, D u, u, x, t\right)=0$ is considered under some continuity assumptions on $F$. We show that the Rothe solutions are Lipschitz in time, Hölder in space, and they solve the equation in the viscosity sense. As an immediate corollary we get Lipschitz behavior in time of the viscosity solutions of our equation.
\end{abstract}

\section{Introduction}

We consider the boundary value problem:

$$
\begin{aligned}
u_{t}+F\left(D_{x}^{2} u, D_{x} u, u, x, t\right) & =0 \quad \text { in } \quad D \\
u & =0 \quad \text { on } \partial_{p} D .
\end{aligned}
$$

We assume that $u(x, t)$ satisfies Equation (1.1) in the viscosity sense, that $D=\Omega \times[0, T]$, and that $F$ is uniformly elliptic. We recall that a function $u$ is a viscosity subsolution (supersolution) of Equation (1.1) if for any $\varphi \in C^{2,1}(\bar{D})$ and any $\epsilon>0$ which satisfies

$$
\begin{aligned}
\varphi_{t} & +F\left(D^{2} \varphi, D \varphi, \varphi, x, t\right) \geq \epsilon>0 \\
\text { (or } \quad \varphi_{t} & +F\left(D^{2} \varphi, D \varphi, \varphi, x, t\right) \leq-\epsilon<0 \text { for the supersolution case) }
\end{aligned}
$$

$u-\varphi$ cannot attain a local maximum (minimum) of 0 . (In other words, $\varphi$ cannot touch $u$ from above (below).) $u$ is a solution if it is both a subsolution and a supersolution. Uniform ellipticity means that there are positive numbers $\lambda$ and $\Lambda$ such that for any positive definite matrix $N$ we have

$$
-\Lambda\|N\| \leq F(M+N, P, v, x, t)-F(M, P, v, x, t) \leq-\lambda\|N\|,
$$

or what is equivalent:

$$
F(M+N, P, v, x, t) \leq F(M, P, v, x, t)+\Lambda\left\|N^{-}\right\|-\lambda\left\|N^{+}\right\|
$$

Math Subject Classifications. 35J60; 65M20.

Key Words and Phrases. Rothe's method, the method of lines, fully nonlinear, parabolic PDE, viscosity solutions.

Acknowledgements and Notes. The first author was partly supported by the NSF grant No. DMS-0501770. 
for all $M$ and $N$. So the negative Laplacian would fit our structure conditions, and not the positive Laplacian.

For linear $F$ 's, Rothe's method is commonly used as a numerical approximation. Rothe's method corresponds to doing a backward Euler approximation in Banach space, and is also known sometimes as the method of lines. Kikuchi and Kačur have studied the convergence properties of Rothe's method in a series of recent articles in a variety of function spaces. (See [5] and [6] and the references therein.) Pluschke has studied the quasilinear case (see [7]), but the current work appears to be the first extension of Rothe's method to viscosity solutions in the fully nonlinear setting.

We fix our mesh size, $0<h \leq 1$, and define $z_{n, h}(x)$ recursively. We take $z_{0, h}(x) \equiv 0$, and for $n \geq 0$ we let $z_{n+1, h}$ be the solution of the elliptic problem:

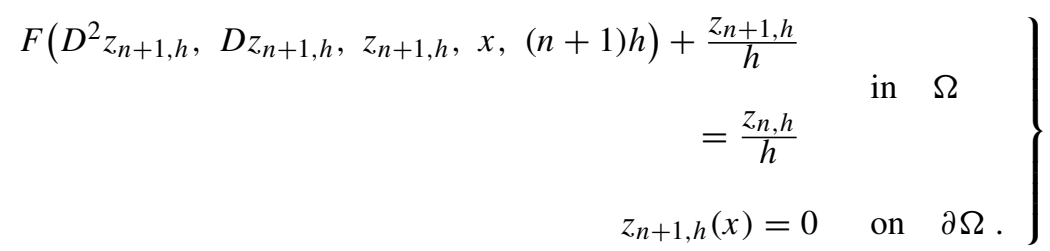

$z_{n, h}(x)$ is an approximation to $u(x, n h)$, and we define the linear interpolation, $U_{h}(x, t)$, by

$$
U_{h}(x, t):=\frac{[(m+1) h-t]}{h} z_{m, h}(x)+\frac{[t-m h]}{h} z_{m+1, h}(x),
$$

where $m \in \mathbb{N}$ is chosen so that $t \in[m h,(m+1) h)$. When $U(x, t):=\lim _{h \downarrow 0} U_{h}(x, t)$ exists, we call it the Rothe limit. Before we show convergence and uniqueness, when a subsequence converges we will still refer to it as a Rothe limit.

We make the following continuity assumption, which corresponds to the structure condition (SC) of [1]: We assume that for every $R>0$, there is a nondecreasing continuous function $\sigma_{R}$ such that $\omega_{R}(0)=0$, and if $X$ and $Y$ are symmetric matrices and $|r|,|s| \leq R$, then

$$
\begin{aligned}
\mathcal{P}^{-} & (M-N)-\gamma|P-Q|-\omega_{R}\left((s-r)^{+}\right) \\
\quad & \leq F(M, P, r, x, t)-F(N, Q, s, x, t) \\
& \leq \mathcal{P}^{+}(M-N)+\gamma|P-Q|+\omega_{R}\left((r-s)^{+}\right) .
\end{aligned}
$$

(We will define the Pucci operators $\mathcal{P}^{+}$and $\mathcal{P}^{-}$in a moment.) Lastly, we assume that for any compact subset of $\mathbb{R}^{n \times n} \times \mathbb{R}^{n} \times \mathbb{R}$ we have

$$
\|F(M, P, r, \cdot, \cdot)\|_{C^{0}(\bar{D})} \leq C<\infty .
$$

\section{Elliptic preliminaries}

To study the elliptic problem in each time we introduce the Pucci extremal operators which we give by

$$
\begin{aligned}
& \mathcal{P}^{+}(M)=-\lambda \operatorname{Tr}\left(M^{+}\right)+\Lambda \operatorname{Tr}\left(M^{-}\right) \quad \text { and } \\
& \mathcal{P}^{-}(M)=-\Lambda \operatorname{Tr}\left(M^{+}\right)+\lambda \operatorname{Tr}\left(M^{-}\right)
\end{aligned}
$$

where $\operatorname{Tr}(M)$ is the trace of $M$.

Lemma 2.1 (First Bound). There exists a constant $C$ which is independent of $h$ such that

$$
\left\|z_{1, h}\right\|_{L^{\infty}} \leq C .
$$


Proof. By symmetry it suffices to show that there exists a constant $C$ such that $z_{1, h}(x) \leq C$. $z_{1, h}$ satisfies

$$
\begin{aligned}
0 & =F\left(D^{2} z_{1, h}, D z_{1, h}, z_{1, h}, x, h\right)+\frac{z_{1, h}}{h} \\
& \geq F\left(D^{2} z_{1, h}, D z_{1, h}, z_{1, h}, x, h\right) \text { on }\left\{z_{1, h}>0\right\}
\end{aligned}
$$

and therefore by Lemma 2.11 of [1] $z_{1, h}$ is also a solution of

$$
\mathcal{P}^{-}\left(D^{2} z_{1, h}\right)-\gamma\left|D z_{1, h}\right|+F\left(0,0, z_{1, h}, x, h\right) \leq 0
$$

on the set where it is positive. By Proposition 2.12 of [1] we now have

$$
z_{1, h} \leq C\left\|\left(-F\left(0,0, z_{1, h}, x, h\right)\right)^{+}\right\|_{L^{n}\left(\Gamma^{+}\left(z_{1, h}\right)\right)},
$$

where $\Gamma^{+}$denotes the upper contact set. Now by using our structure conditions, we see that

$$
-\sigma_{R}\left(\left(-z_{1, h}\right)^{+}\right) \leq F\left(0,0, z_{1, h}, x, h\right)-F(0,0,0, x, h) \leq \sigma_{R}\left(\left(z_{1, h}\right)^{+}\right) .
$$

Let $f(x):=F(0,0,0, x, h)$. Then we see that

$$
\left[-F\left(0,0, z_{1, h}, x, h\right)\right]^{+} \chi_{\left\{z_{1, h}>0\right\}} \leq|f(x)| .
$$

By combining Equations (2.4), (2.5), and (1.8) we are done.

Theorem 2.2 (First Lipschitz Bound). There exists a constant $C$ which is independent of $h$ such that

$$
\left\|z_{1, h}\right\|_{L^{\infty}} \leq C h
$$

Proof. Let the absolute maximum of $z_{1, h}$ be attained at $x_{0}$, and let $\gamma:=z_{1, h}\left(x_{0}\right)$. We have

$$
\gamma=z_{1, h}\left(x_{0}\right)=-h F\left(D^{2} z_{1, h}\left(x_{0}\right), 0, \gamma, x_{0}, h\right)
$$

in the viscosity sense. Since the plane $\Pi(x) \equiv \gamma$ touches $z_{1, h}$ from above at $x_{0}$ we know that

$$
\gamma \leq-h F\left(0,0, \gamma, x_{0}, h\right)
$$

which we know is bounded from above by a constant times $h$ by using the previous lemma along with Equations (1.7) and (1.8).

Theorem 2.3 (Pucci Inequalities). Let $f(x):=F(0,0,0, x,(n+1) h)$. The following inequalities are satisfied in the viscosity sense.

$$
\begin{gathered}
\mathcal{P}^{-}\left(D^{2} z_{n+1}\right)-\gamma\left\|D z_{n+1}\right\|-\sigma_{R}\left(\left(-z_{n+1}\right)^{+}\right)+\frac{z_{n+1}}{h}+f \leq \frac{z_{n}}{h} \\
\leq \mathcal{P}^{+}\left(D^{2} z_{n+1}\right)+\gamma\left\|D z_{n+1}\right\|+\sigma_{R}\left(z_{n+1}^{+}\right)+\frac{z_{n+1}}{h}+f
\end{gathered}
$$

Proof. Let $\varphi$ touch $z_{n+1}$ from above at $x_{0}$. Since

$$
F\left(D^{2} z_{n+1}, D z_{n+1}, z_{n+1}, x,(n+1) h\right)+\frac{z_{n+1}}{h}=\frac{z_{n}}{h}
$$


in the viscosity sense, by using Equation (1.7) we have

$$
\begin{aligned}
\frac{z_{n}}{h} & \geq F\left(D^{2} \varphi, D \varphi, \varphi, x,(n+1) h\right)+\frac{\varphi}{h} \\
& \geq \mathcal{P}^{-}\left(D^{2} \varphi\right)-\gamma\|D \varphi\|-\sigma_{R}\left((-\varphi)^{+}\right)+\frac{\varphi}{h}+f .
\end{aligned}
$$

The other inequality is proven in the same fashion by touching $z_{n+1}$ from below.

Now we introduce the sup-convolution $z_{j}^{\epsilon}$ of $z_{j}$ and the inf-convolution $z_{j, \epsilon}$ of $z_{j}$ which are defined by

$$
z_{j}^{\epsilon}(x):=\sup _{y \in \Omega}\left(z_{j}(y)-\frac{|x-y|^{2}}{2 \epsilon}\right) \quad z_{j, \epsilon}(x):=\inf _{y \in \Omega}\left(z_{j}(y)+\frac{|x-y|^{2}}{2 \epsilon}\right) .
$$

The next two results are taken from [3] and [4], respectively:

Theorem 2.4 (Basic Sup-Convolution Properties). Let $\Omega \subset \mathbb{R}^{n}$ be bounded, let $u \in C(\bar{\Omega})$, and let $u^{\epsilon}$ and $u_{\epsilon}$ be its sup and inf-convolution, respectively. Then

(i) $u^{\epsilon}, u_{\epsilon} \in C^{0,1}(\Omega)$.

(ii) $u^{\epsilon} \downarrow u$ and $u_{\epsilon} \uparrow u$ as $\epsilon \downarrow 0$ uniformly on $\bar{\Omega}$.

(iii) For every $\epsilon>0$, there are measurable functions $M^{\epsilon}, M_{\epsilon}: \Omega \rightarrow \mathcal{S}(n)$ such that

$$
u^{\epsilon}(y)=u^{\epsilon}(x)+<D u^{\epsilon}(x), y-x>+\frac{1}{2}<M^{\epsilon}(x)(y-x), y-x>+o\left(|x-y|^{2}\right)
$$

for a.e. $x \in \Omega$ (and similarly with $u_{\epsilon}$ and $M_{\epsilon}$ ) where $\mathcal{S}(n)$ is the set of $n \times n$ symmetric matrices.

(iv) $M^{\epsilon}(x) \geq-(1 / \epsilon) I$ and $M_{\epsilon}(x) \leq(1 / \epsilon) I$ for a.e $x \in \Omega$.

(v) If $u^{\eta, \epsilon}$ and $u_{\epsilon}^{\eta}$ are standard mollifications of $u^{\epsilon}$ and $u_{\epsilon}$, respectively, then $D^{2} u^{\eta, \epsilon} \geq$ $-(1 / \epsilon) I$ and $D^{2} u^{\eta, \epsilon} \rightarrow M^{\epsilon}(x)$ a.e. $x \in \Omega$ as $\eta \rightarrow 0$, and similarly with $D^{2} u_{\epsilon}^{\eta}$.

Theorem 2.5 (Sup-Convolutions are Subsolutions). If $u$ is a bounded viscosity subsolution of

$$
F\left(D^{2} u, D u, u, x\right)=f(x) \text { in } B_{1}
$$

and $f, F$ are continuous, then

$$
F\left(M^{\epsilon}(x), D u^{\epsilon}(x), u\left(x^{\epsilon}\right), x^{\epsilon}\right) \leq f\left(x^{\epsilon}\right) \quad \text { a.e. in } \quad B_{1-2\left(\epsilon \mid u \|_{\infty}\right)^{1 / 2}}
$$

where $x^{\epsilon} \in B_{1}$ is any point which satisfies

$$
u^{\epsilon}(x)=u\left(x^{\epsilon}\right)-\frac{\left|x^{\epsilon}-x\right|^{2}}{2 \epsilon} .
$$

Obviously inf-convolutions are supersolutions.

Remark 2.6. What we have denoted by " $x^{\epsilon}$," is typically denoted by " $x^{*}$," but we need to emphasize the dependence on $\epsilon$ to avoid confusion later. (For inf-convolutions we will use " $x_{\epsilon}$.")

Remark 2.7. Note that

$$
\frac{\left|x^{\epsilon}-x\right|^{2}}{2 \epsilon}=u^{\epsilon}\left(x^{\epsilon}\right)-u^{\epsilon}(x) \leq 2\|u\|_{\infty},
$$


so that $\left|x^{\epsilon}-x\right| \leq 2\left(\epsilon\|u\|_{\infty}\right)^{1 / 2}$. In particular, if $x \in B_{1-2\left(\epsilon|| u \|_{\infty}\right)^{1 / 2}}$ then $x^{\epsilon} \in B_{1}$, explaining the appearance of this set above.

By using Equation (1.5) and the previous theorem, we get

$$
F\left(M_{n+1, h}^{\epsilon}(x), D z_{n+1, h}^{\epsilon}(x), z_{n+1, h}^{\epsilon}\left(x^{\epsilon}\right), x^{\epsilon},(n+1) h\right)+\frac{z_{n+1, h}^{\epsilon}\left(x^{\epsilon}\right)}{h} \leq \frac{z_{n, h}\left(x^{\epsilon}\right)}{h}
$$

for a.e. $x$. Similarly, we have

$$
F\left(M_{n, h, \epsilon}(x), D z_{n, h, \epsilon}(x), z_{n, h, \epsilon}\left(x_{\epsilon}\right), x_{\epsilon}, n h\right)+\frac{z_{n, h, \epsilon}\left(x_{\epsilon}\right)}{h} \geq \frac{z_{n-1, h}\left(x_{\epsilon}\right)}{h} .
$$

Henceforth we suppress the $h$ in the subscripts. By taking a difference we get:

$$
\begin{aligned}
F( & \left.M_{n+1}^{\epsilon}(x), D z_{n+1}^{\epsilon}(x), z_{n+1}^{\epsilon}\left(x^{\epsilon}\right), x^{\epsilon},(n+1) h\right) \\
& \quad-F\left(M_{n, \epsilon}(x), D z_{n, \epsilon}(x), z_{n, \epsilon}\left(x_{\epsilon}\right), x_{\epsilon}, n h\right)+\frac{z_{n+1}^{\epsilon}\left(x^{\epsilon}\right)-z_{n, \epsilon}\left(x_{\epsilon}\right)}{h} \\
\leq & \frac{z_{n}\left(x^{\epsilon}\right)-z_{n-1}\left(x_{\epsilon}\right)}{h} .
\end{aligned}
$$

Now we express the difference as a telescoping sum.

$$
\begin{array}{r}
F\left(M_{n+1}^{\epsilon}(x), D z_{n+1}^{\epsilon}(x), z_{n+1}^{\epsilon}\left(x^{\epsilon}\right), x^{\epsilon},(n+1) h\right) \\
-F\left(M_{n, \epsilon}(x), D z_{n, \epsilon}(x), z_{n, \epsilon}\left(x_{\epsilon}\right), x_{\epsilon}, n h\right) \\
=F\left(M_{n+1}^{\epsilon}(x), D z_{n+1}^{\epsilon}(x), z_{n+1}^{\epsilon}\left(x^{\epsilon}\right), x^{\epsilon},(n+1) h\right) \\
-F\left(M_{n, \epsilon}(x), D z_{n+1}^{\epsilon}(x), z_{n+1}^{\epsilon}\left(x^{\epsilon}\right), x^{\epsilon},(n+1) h\right) \\
+F\left(M_{n, \epsilon}(x), D z_{n+1}^{\epsilon}(x), z_{n+1}^{\epsilon}\left(x^{\epsilon}\right), x^{\epsilon},(n+1) h\right) \\
-F\left(M_{n, \epsilon}(x), D z_{n, \epsilon}(x), z_{n+1}^{\epsilon}\left(x^{\epsilon}\right), x^{\epsilon},(n+1) h\right) \\
+F\left(M_{n, \epsilon}(x), D z_{n, \epsilon}(x), z_{n+1}^{\epsilon}\left(x^{\epsilon}\right), x^{\epsilon},(n+1) h\right) \\
-F\left(M_{n, \epsilon}(x), D z_{n, \epsilon}(x), z_{n, \epsilon}\left(x_{\epsilon}\right), x^{\epsilon},(n+1) h\right) \\
+F\left(M_{n, \epsilon}(x), D z_{n, \epsilon}(x), z_{n, \epsilon}\left(x_{\epsilon}\right), x^{\epsilon},(n+1) h\right) \\
\quad-F\left(M_{n, \epsilon}(x), D z_{n, \epsilon}(x), z_{n, \epsilon}\left(x_{\epsilon}\right), x_{\epsilon}, n h\right) .
\end{array}
$$

For the time being we will assume that $F$ is differentiable in the variables we need in order to define

$$
\begin{aligned}
a_{i j}(x):= & \int_{0}^{1} F_{X_{i j}}\left(t M_{n+1}^{\epsilon}(x)+(1-t) M_{n, \epsilon}(x),\right. \\
& \left.D z_{n+1}^{\epsilon}(x), z_{n+1}^{\epsilon}\left(x^{\epsilon}\right), x^{\epsilon},(n+1) h\right) d t \\
b_{i}(x):= & \int_{0}^{1} F_{P_{i}}\left(M_{n, \epsilon}(x), t D z_{n+1}^{\epsilon}(x)+(1-t) D z_{n, \epsilon}(x),\right. \\
& \left.z_{n+1}^{\epsilon}\left(x^{\epsilon}\right), x^{\epsilon},(n+1) h\right) d t \\
\tilde{c}(x):= & \int_{0}^{1} F_{r}\left(M_{n, \epsilon}(x), D z_{n, \epsilon}(x), t z_{n+1}^{\epsilon}\left(x^{\epsilon}\right)\right. \\
& \left.+(1-t) z_{n, \epsilon}\left(x_{\epsilon}\right), x^{\epsilon},(n+1) h\right) d t .
\end{aligned}
$$

Now we use the fundamental theorem on each pair of terms to get the following equalities:

$$
\begin{aligned}
& F\left(M_{n+1}^{\epsilon}(x), D z_{n+1}^{\epsilon}(x), z_{n+1}^{\epsilon}\left(x^{\epsilon}\right), x^{\epsilon},(n+1) h\right) \\
& \quad-F\left(M_{n, \epsilon}(x), D z_{n+1}^{\epsilon}(x), z_{n+1}^{\epsilon}\left(x^{\epsilon}\right), x^{\epsilon},(n+1) h\right) \\
& =a_{i j}(x)\left(M_{n+1,(i j)}^{\epsilon}(x)-M_{n, \epsilon,(i j)}(x)\right)
\end{aligned}
$$




$$
\left.\begin{array}{rl}
F & \left(M_{n, \epsilon}(x), D z_{n+1}^{\epsilon}(x), z_{n+1}^{\epsilon}\left(x^{\epsilon}\right), x^{\epsilon},(n+1) h\right) \\
& -F\left(M_{n, \epsilon}(x), D z_{n, \epsilon}(x), z_{n+1}^{\epsilon}\left(x^{\epsilon}\right), x^{\epsilon},(n+1) h\right) \\
= & b_{i}(x) D_{i}\left(z_{n+1}^{\epsilon}(x)-z_{n, \epsilon}(x)\right) \\
& F\left(M_{n, \epsilon}(x), D z_{n, \epsilon}(x), z_{n+1}^{\epsilon}\left(x^{\epsilon}\right), x^{\epsilon},(n+1) h\right) \\
& -F\left(M_{n, \epsilon}(x), D z_{n, \epsilon}(x), z_{n, \epsilon}\left(x_{\epsilon}\right), x^{\epsilon},(n+1) h\right) \\
& =\tilde{c}(x)\left(z_{n+1}^{\epsilon}\left(x^{\epsilon}\right)-z_{n, \epsilon}\left(x_{\epsilon}\right)\right) .
\end{array}\right\}
$$

By the continuity assumption (1.8) we also know

$$
\begin{aligned}
& \mid F\left(M_{n, \epsilon}(x), D z_{n, \epsilon}(x), z_{n, \epsilon}\left(x_{\epsilon}\right), x^{\epsilon},(n+1) h\right) \\
& \quad-F\left(M_{n, \epsilon}(x), D z_{n, \epsilon}(x), z_{n, \epsilon}\left(x_{\epsilon}\right), x_{\epsilon}, n h\right) \mid \\
& \quad \leq \sigma_{1}(\epsilon)+\sigma_{2}(h)
\end{aligned}
$$

where the $\sigma_{i}$ are moduli of continuity. By defining $c(x):=\tilde{c}(x)+(1 / h), w_{n}^{\epsilon}(x):=z_{n+1}^{\epsilon}(x)-$ $z_{n, \epsilon}(x)$, by defining $D_{i j} w_{n}^{\epsilon}:=M_{n+1}^{\epsilon}-M_{n, \epsilon}(x)$, and by using the equations beginning with Equation (2.12) we can conclude

$$
\begin{aligned}
& a_{i j}(x) D_{i j} w_{n}^{\epsilon}(x)+b_{i}(x) D_{i} w_{n}^{\epsilon}(x)+c(x)\left[w_{n}^{\epsilon}\left(x^{\epsilon}\right)+z_{n, \epsilon}\left(x^{\epsilon}\right)-z_{n, \epsilon}\left(x_{\epsilon}\right)\right] \\
& \quad \leq \frac{z_{n}\left(x^{\epsilon}\right)-z_{n-1}\left(x_{\epsilon}\right)}{h}+\sigma_{1}(\epsilon)+\sigma_{2}(h) \text { for } \text { a.e. } x .
\end{aligned}
$$

We define a semiconvex function to be a function which becomes convex if $\gamma\|x\|^{2}$ is added to the function, and $\gamma$ is sufficiently large. In particular, by Theorem 2.4 (iv) we know that sup-convolutions are semiconvex, as is the function $w_{n}^{\epsilon}$ in Equation (2.19). Now we need the following results (see p. 56 Theorem A.2 and p. 58 Lemma A.3 of [2]).

Theorem 2.8 (Aleksandrov's Theorem). Semiconvex functions are twice differentiable a.e.

Lemma 2.9 (Jensen's Lemma). Let $\varphi: \mathbb{R}^{n} \rightarrow \mathbb{R}$ be semiconvex, and let $\hat{x}$ be a strict local maximum of $\varphi$. For $p \in \mathbb{R}^{n}$, set $\varphi_{p}(x)=\varphi(x)+<p, x>$. Then for any $r, \delta>0$, the set

$$
K:=\left\{x \in B_{r}(\hat{x}): \text { there exists } p \in B_{\delta} \text { such that } \varphi_{p} \text { has a local max at } x\right\}
$$

has positive measure.

We also state for future use the following estimate.

Lemma 2.10 (Discrete Gronwall Inequality). Assuming $\left\{v_{i}\right\},\left\{B_{i}\right\}$, and $\left\{D_{i}\right\}$ are sequences of nonnegative numbers which satisfy $v_{i+1} \leq B_{i} v_{i}+D_{i}$, we have

$$
v_{n} \leq v_{0} \prod_{i=0}^{n-1} B_{i}+\sum_{i=0}^{n-1}\left[D_{i} \prod_{j=i+1}^{n-1} B_{j}\right] .
$$

With these results we can turn to the estimate of the maximum of $w_{n}(x):=z_{n+1}(x)-z_{n}(x)$.

\section{Rothe limits are Lipschitz in time}

Theorem 3.1 (Inductive Estimation). If $F$ satisfies (1.7), then we have

$$
\left\|w_{n}\right\|_{L^{\infty}(\Omega)} \leq\left\|w_{n-1}\right\|_{L^{\infty}(\Omega)}+h \sigma_{2}(h) .
$$


Furthermore, if we assume that $\sigma_{2}(h) \leq C h$, then for any fixed $T>0$, we have the Lipschitz estimate:

$$
\left\|\frac{w_{[T / h]}}{h}\right\|_{L^{\infty}(\Omega)} \leq C,
$$

where $C$ is independent of $h$, and $[T / h]$ is the greatest integer less than or equal to $T / h$.

Proof. We will first prove Equation (3.1). To start we will need a few assumptions: First that $F$ is differentiable so that Equation (2.19) is valid, second that $w_{n}$ has a strict maximum at $\hat{x}$, and finally that our approximating functions, $w_{n}^{\epsilon}$ are twice differentiable at their corresponding maxima which we call $\hat{x}_{\epsilon}$. We will show how to avoid these assumptions after proving the estimate in this case.

Because $\hat{x}$ is a maximum, and $w_{n}^{\epsilon}$ is twice differentiable at $\hat{x}^{\epsilon}$, Equation (2.19) becomes:

$$
c(\hat{x})\left[w_{n}^{\epsilon}\left(\hat{x}^{\epsilon}\right)+z_{n, \epsilon}\left(\hat{x}^{\epsilon}\right)-z_{n, \epsilon}\left(\hat{x}_{\epsilon}\right)\right] \leq \frac{z_{n}\left(\hat{x}^{\epsilon}\right)-z_{n-1}\left(\hat{x}_{\epsilon}\right)}{h}+\sigma_{1}(\epsilon)+\sigma_{2}(h) .
$$

Now by using Remark 2.7 we see that

$$
\left|\hat{x}^{\epsilon}-\hat{x}_{\epsilon}\right| \leq 2\left(\epsilon\left\|z_{n+1}^{\epsilon}\right\|_{\infty}\right)^{1 / 2}+2\left(\epsilon\left\|z_{n, \epsilon}\right\|_{\infty}\right)^{1 / 2},
$$

and then by sending $\epsilon$ to zero and using the Hölder regularity of the $z_{n}$ in space we can conclude:

$$
\tilde{c}(\hat{x}) w_{n}(\hat{x})+\frac{w_{n}(\hat{x})}{h}=c(\hat{x}) w_{n}(\hat{x}) \leq \frac{w_{n-1}(\hat{x})}{h}+\sigma_{2}(h) .
$$

By using Equation (1.7) we see that $F_{r} \geq 0$ and therefore $\tilde{c} \geq 0$. So, since $w_{n}(\hat{x}) \geq 0$, we have

$$
w_{n}(\hat{x}) \leq\left\|w_{n-1}\right\|_{L^{\infty}(\Omega)}+h \sigma_{2}(h) .
$$

Obviously we can argue similarly for a negative minimum, but it remains to show how to eliminate our regularity assumptions. First of all we need stability of our approximations as we take smooth approximators $F_{m}$, to our original $F$. The desired stability can be found in Section 6 of [2]. (See Remark 6.3 in [2] in particular.) In terms of applying the estimates we conclude for our $F_{m}$ 's for our original $F$ there is no problem, because no matter how badly behaved the $a_{i j}, b_{i}$, and $\tilde{c}$ are in a given approximation to $F$, they get thrown away at the maximum of $w_{n}$ by considerations which depend only on the signs of the terms involved. Stated differently, even if the derivatives of the approximations are diverging, they are necessarily diverging "in the right direction." (Equation (3.3) is obtained from Equation (2.19) by using the nonpositivity of the second derivative and the vanishing of the gradient of $w_{n}$ at its maximum.)

Next, if $w_{n}$ has a maximum at $\hat{x}$ but it is not the unique maximum, then we look at the equation satisfied by $\tilde{w}_{n}(x):=w_{n}(x)-\hat{\epsilon}|x-\hat{x}|^{2}$, where $\hat{\epsilon}>0$ is arbitrarily small. (Here we need the continuity assumptions on our function $F$ given in (1.7).) Finally, to ensure twice differentiability of the $w_{n}^{\epsilon}$ at $\hat{x}^{\epsilon}$ we appeal to Jensen's Lemma 2.9 and Aleksandrov's Theorem 2.8.

Now we need to prove Equation (3.2). We apply Lemma 2.10 to Equation (3.1) to get

$$
\left\|w_{n}\right\|_{L^{\infty}} \leq\left\|w_{0}\right\|_{L^{\infty}}+n h \sigma_{2}(h)
$$

with $n=[T / h]$ and by using Theorem 2.2 we are done.

Theorem 3.2 (Existence of Rothe Limits). If $\sigma_{2}(h) \leq C h$ then the Rothe limit exists after choosing an appropriate subsequence, and these limits are locally Lipschitz in time, and locally Hölder in space. 
Remark 3.3 (Uniqueness of the Rothe Limit). In fact, we will be able to deduce uniqueness of the Rothe limit and convergence along the original sequence without taking subsequences by the results of the next section.

Proof. The last theorem shows that the sequence of approximators are uniformly Lipschitz in time. In order to show that the approximators are equicontinuous in space, we observe that our uniform Lipschitz estimate implies $F\left(D^{2} z_{n+1}, D z_{n+1}, z_{n+1}, x,(n+1) h\right)$ is uniformly bounded in $L^{\infty}$ in the viscosity sense. Then we invoke the spatial Hölder estimates known for fully nonlinear elliptic equations (see Theorem 5.1 Part III of [9]). Finally, we can invoke Arzela-Ascoli to guarantee the existence of the limit after choosing a subsequence.

\section{Rothe limits are viscosity solutions}

Theorem 4.1 (Rothe Limits Are Viscosity Solutions). The Rothe limits are viscosity solutions.

Proof. By symmetry, it will suffice to prove that Rothe limits are viscosity subsolutions. Suppose not. Then there exists an $\epsilon>0$ and a supersolution $\varphi \in C^{2,1}(\bar{D})$ which touches a given Rothe limit $U$ from above at a point $\left(x_{0}, t_{0}\right)$ and which satisfies:

$$
\varphi_{t}+F\left(D^{2} \varphi, D \varphi, \varphi, x, t\right) \geq \epsilon>0,
$$

pointwise in a neighborhood of $\left(x_{0}, t_{0}\right)$. Now let $H$ be the set of $h$ which belong to the subsequence which converges to $U$. By adding a very small multiple of $\left|t-t_{0}\right|^{2}+\left|x-x_{0}\right|^{2}$ to $\varphi$, by using the continuity of $F$, and by allowing a slightly smaller (but still positive) $\epsilon$ in Equation (4.1), we can assume without loss of generality that at least for a small neighborhood of $\left(x_{0}, t_{0}\right)$ which we will call $N$ the only contact between $\varphi$ and $U$ is at $\left(x_{0}, t_{0}\right)$. Because of the uniform continuity of $\varphi_{t}$ there exists a neighborhood $\tilde{N}$ of $\left(x_{0}, t_{0}\right)$ such that for a sufficiently small $\tilde{h}>0$, and for all $(x, t) \in \tilde{N}$ we have the estimate

$$
\left|\frac{\varphi(x, t)-\varphi(x, t-h)}{h}-\varphi_{t}(x, t)\right| \leq \frac{\epsilon}{10}
$$

for all $h \leq \tilde{h}$, with $h \in H$. (For the remainder of this proof we will abuse notation by not mentioning that $h$ always belongs to $H$.) At this point let $\mathcal{N}$ denote a compact set of the form $\overline{B_{r}\left(x_{0}\right)} \times\left[t_{0}-\gamma, t_{0}+\gamma\right]$ which is contained in $N \cap \tilde{N}$. Let

$$
\mathcal{S}:=\mathcal{N} \backslash\left\{B_{r / 2}\left(x_{0}\right) \times\left(t_{0}-\gamma / 2, t_{0}+\gamma / 2\right)\right\} .
$$

Because $\varphi>U$ on $\mathcal{S}$, there exists a $\tilde{\epsilon}>0$ such that $\varphi \geq U+\tilde{\epsilon}$ on $\mathcal{S}$. By the uniform convergence of $U_{h}$ to $U$, for any $\delta \in(0, \tilde{\epsilon} / 3)$ we can be sure that by shrinking $\tilde{h}$ if necessary we have

$$
\varphi>U_{h}+\tilde{\epsilon}-\delta \text { on } \mathcal{S} \text { while }\left|\varphi\left(x_{0}, t_{0}\right)-U_{h}\left(x_{0}, t_{0}\right)\right|<\delta / 4
$$

for all $h \leq \tilde{h}$. Choose $\delta$ sufficiently small to guarantee that

$$
\omega_{R}(\delta)<\epsilon / 2,
$$

where $\omega_{R}$ is the modulus given in Equation (1.7) and $\epsilon$ is taken from Equation (4.1).

Fix $h<\min \{\tilde{h}, \gamma / 10\}$, and sufficiently small to guarantee that

$$
\left|\varphi\left(x_{0}, t\right)-U_{h}\left(x_{0}, t\right)\right|<\delta / 2
$$


for any $t \in\left[t_{0}-h, t_{0}\right]$. (Here we need the second half of Equation (4.4) and uniform continuity in time of $\varphi\left(x_{0}, t\right)-U_{h}\left(x_{0}, t\right)$ as $h \downarrow 0$. The continuity is guaranteed by the previous theorem.) If we let $\tilde{\varphi}=\varphi+c$, for any $c$ with $|c|<\delta$, then by using Equations (1.7) and (4.5) we will have

$$
\tilde{\varphi}_{t}+F\left(D^{2} \tilde{\varphi}, D \tilde{\varphi}, \tilde{\varphi}, x, t\right) \geq \epsilon-\omega_{R}(\delta)>\epsilon / 2>0 .
$$

Now we choose $c$ so that the minimum of $\tilde{\varphi}-U_{h}$ on the set $\mathcal{N} \cap\{(x, t): t \in \mathbb{N} h\}$ is equal to zero. In other words, $\tilde{\varphi}$ is allowed to be less than $U_{h}$ between the mesh values of $t$, but when considering only mesh values of $t$, we can say that $\tilde{\varphi}$ touches $U_{h}$ from above.

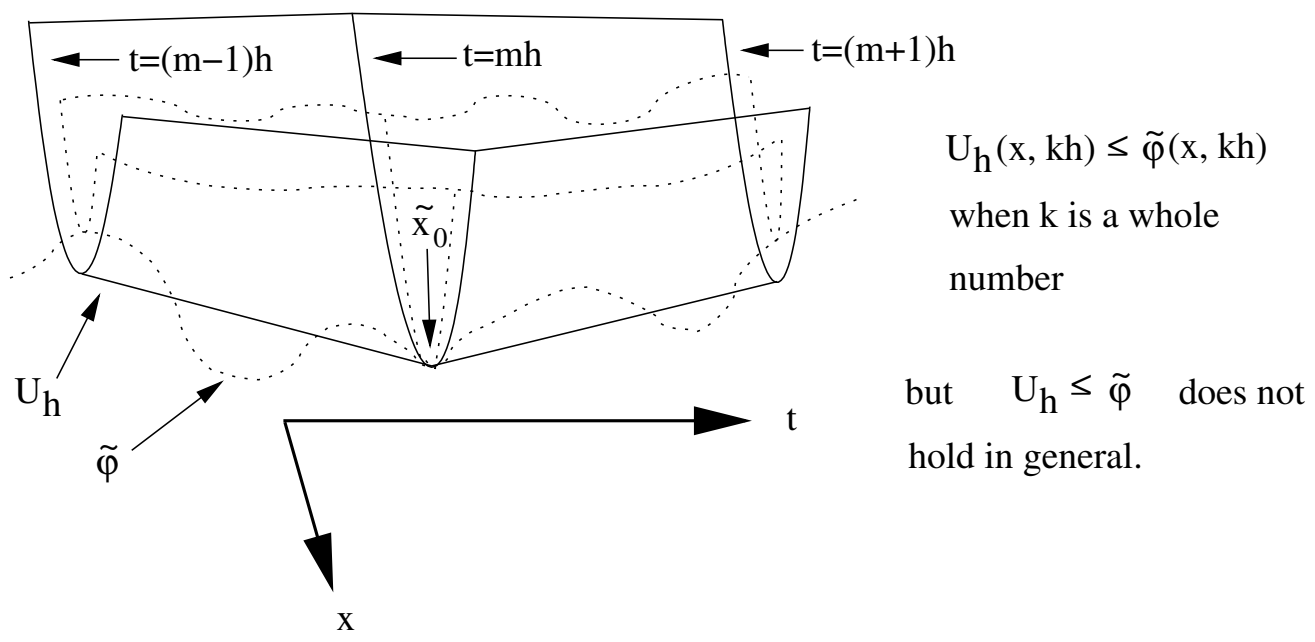

FIGURE 1

Because of Equation (4.6), we can be sure that $|c| \leq \delta / 2$. Let $\left(\tilde{x_{0}}, m h\right)$ be a point where $\tilde{\varphi}=U_{h}$. By using Equation (4.4) we know that $\left(\tilde{x_{0}}, m h\right) \in B_{r / 2}\left(x_{0}\right) \times\left(t_{0}-\gamma / 2, t_{0}+\gamma / 2\right)$.

If we return to our $z_{n}$ we now have the following setting:

(a) $\tilde{\varphi}(x, m h)$ touches $z_{m}(x)$ from above at $\tilde{x_{0}}$.

(b) $\tilde{\varphi}(x,(m-1) h) \geq z_{m-1}(x)$ in $B_{r / 2}\left(x_{0}\right)$. (Because $h<\gamma / 10$ we know that $B_{r / 2}\left(x_{0}\right) \times$ $\{t=(m-1) h\} \subset \mathcal{N}$.)

Because $\left(\tilde{x_{0}}, m h\right) \subset \tilde{N}$ we have [see Equation (4.2)]

$$
\left|\frac{\tilde{\varphi}\left(\tilde{x_{0}}, m h\right)-\tilde{\varphi}\left(\tilde{x_{0}},(m-1) h\right)}{h}-\tilde{\varphi}_{t}\left(\tilde{x_{0}}, m h\right)\right| \leq \frac{\epsilon}{10} .
$$

Recall that $z_{m}$ is a viscosity solution of

$$
\frac{z_{m}-z_{m-1}}{h}+F\left(D^{2} z_{m}, D z_{m}, z_{m}, x, m h\right)=0 .
$$

Since $\tilde{\varphi}(x, m h)$ touches $z_{m}(x)$ from above at $\tilde{x_{0}}$, we conclude that

$$
\frac{\tilde{\varphi}-z_{m-1}\left(\tilde{x_{0}}\right)}{h}+F\left(D^{2} \tilde{\varphi}, D \tilde{\varphi}, \tilde{\varphi}, \tilde{x_{0}}, m h\right) \leq 0 .
$$


Now we combine Equations (4.7), (4.10), and (4.8) to get

$$
\begin{aligned}
\epsilon / 2 & <\tilde{\varphi}_{t}\left(\tilde{x_{0}}\right)+F\left(D^{2} \tilde{\varphi}, D \tilde{\varphi}, \tilde{\varphi}, \tilde{x_{0}}, m h\right) \\
& \leq \tilde{\varphi}_{t}\left(\tilde{x_{0}}\right)+\frac{z_{m-1}\left(\tilde{x_{0}}\right)-\tilde{\varphi}\left(\tilde{x_{0}}, m h\right)}{h} \\
& \leq \tilde{\varphi}_{t}\left(\tilde{x_{0}}\right)+\frac{\tilde{\varphi}\left(\tilde{x_{0}},(m-1) h\right)-\tilde{\varphi}\left(\tilde{x_{0}}, m h\right)}{h} \\
& \leq \epsilon / 10
\end{aligned}
$$

which is a contradiction.

Now by invoking Jensen's uniqueness theorem for viscosity solutions we get the following corollary.

Corollary 4.2 (The Rothe Limit Exists and is Unique). The Rothe limit exists and is unique.

Corollary 4.3 (Lipschitz Regularity in Time). The viscosity solution to Equation (1.1) is Lipschitz in time.

Proof. Simply combine the three previous results.

\section{References}

[1] Caffarelli, L. A., Crandall, M. G., Kocan, M., and Święch, A. On viscosity solutions of fully nonlinear equations with measurable ingredients, Comm. Pure Appl. Math. 49(4), 365-397, (1996).

[2] Crandall, M. G., Ishii, H., and Lions, P.-L. User's guide to viscosity solutions of second order partial differential equations, Bull. Amer. Math. Soc. 27(1), 1-67, (1992).

[3] Jensen, R. The maximum principle for viscosity solutions of fully nonlinear second order partial differential equations, Arch. Ration. Mech. Anal. 101, 1-27, (1988).

[4] Jensen, R., Lions, P.-L., and Souganidis, P. E. A uniqueness result for viscosity solutions of second order fully nonlinear partial differential equations, Proc. Amer. Math. Soc. 102, 975-978, (1988).

[5] Kikuchi, N. Hölder estimates of solutions to difference partial differential equations of elliptic-parabolic type, J. Geom. Anal. 11(1), 77-89, (2001).

[6] Kikuchi, N. and Kačur, J. Convergence of Rothe's method in Hölder spaces, Appl. Math. 48(5), 353-365, (2003).

[7] Pluschke, V. Local solutions to quasilinear parabolic equations without growth restrictions, Z. Anal. Anwendungen 15, 375-396, (1996).

[8] Rektorys, K. The Method of Discretization in Time and Partial Differential Equations, D. Reidel, (1982).

[9] Trudinger, N. S. Comparison principles and pointwise estimates for viscosity solutions of nonlinear elliptic equations, Rev. Mat. Iberoamericana 4(3/4), 453-468, (1988).

Received June 2, 2004

Department of Mathematics, Worcester Polytechnic Institute, Worcester, MA 01609

e-mail: blanki@wpi.edu

Department of Mathematics, Lehigh University, Bethlehem, PA 18015

e-mail: Penny314@aol.com

Communicated by David Jerison 